\title{
Propuesta para la enseñanza del cuidado en Enfermería
}

\section{Nursing care teaching proposal}

Mtra. Martha Lilia Bernal Becerril* y Mtra. Gandhy Ponce Gómez**.

*Profesor de Carrera Asociado B. de T.C. Unidad de Investigación. Escuela Nacional

de Enfermería y Obstetricia. UNAM y **Profesor de Carrera Asociado B. de T.C.

Unidad de Investigación. Escuela Nacional de Enfermería y Obstetricia. UNAM

\section{Resumen}

La enseñanza en Enfermería ha sido edificada a través de una metodología participativa y de construcción constante, ejerciendo a dos niveles de actuación, uno como formadores de profesionales y el segundo como agente cuidador del individuo, grupo o familia. De manera que a partir de reconocer la naturaleza y complejidad humana, los profesionales en Enfermería son cruciales desde que inicia su formación educativa. Educar a las enfermeras implica entender las interacciones culturales de las cuales ellas son parte.

La verdadera proveedora de cuidado cuidador debe buscar libertad, diálogo, creatividad, autodeterminación y dignidad humana, por lo tanto, es fundamental que la enseñanza

Nursing teaching has been built trough participative methodologies. It acts as a professional former, but also as a care agent. So, considering the complexity of human nature, nursing professionals are crucial; even during their formation years. Educating nurses implies understanding the culture interactions which they are part of.

The true care-giver should pursue freedom, dialog, creativity, self-determination, and human dignity; therefore, it is fundamental that nursing teaching be integral, active, and interactive, so that it leads to the development of values, knowledge, and en Enfermería sea integral, activa, e integrativa, de tal forma que lleve al desarrollo de valores, conocimiento, y relaciones sociales para formar seres humanos que cuiden seres humanos. Para mantener la mejoría en la practica de la enseñanza en Enfermería, en este articulo sugerimos un adecuado uso de los textos y de los contextos, resaltando la importancia del acercamiento al aprendizaje significativo de Ausubel, así como las experiencias previas de los alumnos, para transformar la practica educativa

Palabras clave: Enseñanza del cuidado, texto, contexto

\section{Abstract}

social relations to form human beings to take care of human beings.

In order to keep improving the nursing teaching practice, in this article we suggest an adequate use of texts within the corresponding contexts, highlighting the importance of Ausubel's significant learning approach and the student's previous experiences for transformer the educative practice

Keywords Care teaching, text, Context 


\section{INTRODUCCION}

En cuanto al cuidado, en la formación y práctica de Enfermería había brillado por su ausencia la influencia hermenéutica y fenomenológica hasta finales del siglo XX, incluso, es retomada por Moreno, Muñoz y Alvis ${ }^{1}$, cuando mencionan que "cuidar" implica para Enfermería un conocimiento propio de cada enfermo, un darse cuenta de sus actitudes, aptitudes, intereses y motivaciones y además de conocimientos, que requiere el reconocimiento de su manifestación como persona única, auténtica, capaz de generar confianza, serenidad, seguridad y apoyo humanos.

Para tener la disposición de cuidar se requiere de muchos atributos, que Piccard (1995), Dillon y Wright (1996), Stricland (1996), Fry, Killen y Robinson (1996), han reconocido, entre los que se encuentran: promover el bienestar, empoderamiento, toma de decisiones proactivas, competencias, confianza, conciencia, compromiso, reflexión sobre los valores, principios profesionales y personales expresados en virtudes, actitudes, ideales, conductas y destrezas dentro de un proceso, que además esta circundado por la empatía 1 .

Cuidar, por lo tanto, implica al parecer una complejidad de valores particularmente humanos, pero por el hecho de serlo, se sabe que existe la posibilidad de trascender a este tipo de actuación, en el que sólo hace falta que el enfermero lo desee, lo viva, lo quiera y lo necesite. Implica considerar dimensiones emocionales y morales de salud, no sólo de enfermedad, teniendo en este vínculo empático con el paciente, la oportunidad de profundizar en el alcance y las raíces de la dignidad humana.

Para adentrarnos en este tipo de cuidado, es imprescindible considerar que con los esquemas positivistas en que hemos sido formados casi nuestra generalidad, no se puede llegar al cuidado trascendente, ya que las simples explicaciones, evidencias, suposiciones, elaboraciones supuestamente reales, no dan cabida al fenómeno del cuidado por la complejidad que hemos inferido. Wright ${ }^{2}$, considera, que Enfermería por ser parte de las ciencias sociales, debe hacer uso de la comprensión, donde es necesario entender la intencionalidad de una interacción, profundizar en ritos y creencias de salud, y experiencias en las que se vive esta en forma individual y colectiva.

Por lo tanto, si reconocemos que el concepto de cuidado denota fenómenos complejos, multiculturales y de múltiples facetas, y que además posee elementos científicos y técnicos, es que no podemos prescindir de todos los factores humanísticos aquí mencionados. Que las formas de estu- diar y profundizar en el cuidado, no son solo metodologías positivistas, ya que si se ha echado mano de marcos conceptuales fenomenológicos existenciales y de la psicología humanista, es por tanto obvio y necesario que éstos reconocimientos tengan repercusiones en la forma de enseñar y aprender el cuidado.

\section{EL CUIDADO COMO EJE DE LA ENSEÑANZA EN ENFERMERIA}

Educar según González³, no implica sólo socializar, sino introducirse en el significado de la cultura en la cual esta inmerso el educando, contextualizarse en sus propias estructuras especiales, en ese sentido, la filosofía de educar y la filosofía del cuidado son una misma. En la educación se pretende, desde los postulados constructivistas que el conocimiento no sea una copia fiel de la realidad sino una construcción del ser humano desde el vínculo de los aspectos cognitivos con los aspectos sociales del comportamiento. Se debe construir en función de lo que construyó previamente en su relación con el medio que le rodea. Al tiempo que se pretende generar afiliación por el objeto de estudio en Enfermería, que también sea construida tanto individual como colectivamente este constructo, rotundamente humano y por tanto social.

Los aspectos cognitivos con los aspectos comportamentales aunado a lo que Habermas afirma en el sentido de qué es lo cotidiano de los intereses hacia la parte cognitiva, junto con lo postulado por Husserl que le da importancia a la subjetividad por darle sentido a la experiencia cotidiana. Nos plantean que los intereses particulares e individuales son orientadores de la actividad cognoscitiva y por tanto la reflexión del conocimiento ${ }^{4}$. Las experiencias generadoras o motivantes dentro del proceso enseñanza - aprendizaje en Enfermería no tienen menor trascendencia que las de cualquier otra disciplina profesional. Por lo que es importante desarrollar la concepción de cuidado como objeto de estudio y que en torno a él es que debe girar la experiencia educativa.

Además, los profesionales de la Enfermería son un grupo social que cumple con dos tareas primordiales: el cuidado de los pacientes y la enseñanza de la Enfermería; en donde ambas requieren de un compromiso moral con las necesidades de la sociedad. Ya Leninger (1981) y Watson (1985), reconocen al cuidado como la esencia de Enfermería ${ }^{1}$. Incluso para Medina, la Enfermería es una ciencia humana práctica que usa las teorías de las ciencias naturales y de la conducta para comprender y desarrollar las prácticas del cuidado. El cuidar es esencia de la disciplina, siendo núcleo 
y por tanto objeto de estudio, debe de ser discernido como tal, pero además reconocido por todo profesional de Enfermería que se precie de serlo, y ejercer la Enfermería con vocación, deber y responsabilidad humana ${ }^{5}$.

A pesar de que todavía nos sigue el fantasma del servilismo, la honorabilidad, la moralidad y la humildad que se dieron a Enfermería por la influencia del cristianismo, es también válido reconocer que cuidar es una actitud humana, ya que es en el conocimiento de la persona como ser humano total y completo, y que vive el cuidado de forma particular, que las actividades de cuidar son multifacéticas. Concordamos con Pinto ${ }^{6}$, en decir que las actividades que se realicen en la práctica, y que estén concebidas para orientar la formación disciplinar de la Enfermería, deben tener como foco y dominio que las personas vivan y crezcan en el cuidado.

Los docentes de Enfermería de hoy, deben de estar pensando las formas o caminos de afianzar en el estudiante la confianza, la esperanza, el coraje, la paciencia, la continuidad, la constancia que le dan la oportunidad de establecer no sólo un puente de comunicación, sino un vínculo indisoluble que haga crecer a ambos (docente -alumno, cuidador - sujeto del cuidado) en la experiencia del cuidado para sí mismos, sin transgredir al otro.

De acuerdo Coraggio ${ }^{7}$, para transformar el proceso enseñanza -aprendizaje se deben cumplir con dos puntos; el primero es crear las condiciones más favorables para un aprendizaje efectivo, capaz de desarrollar simultáneamente a educandos y educadores. (uso adecuado del TEXTO con estrategias del aprendizaje significativo). Y segundo, que el aprendizaje no debe restringirse al ámbito del aula, ya que las experiencias extraescolares, contribuyen enormemente a forjar expectativas al momento de incorporarse en el ámbito laboral, y para ello es importante su Motivación (Contexto)

Propuesta Para La Transformación De La Enseñanza Del Cuidado A Partir Del Aprendizaje Significativo Su Uso En El Texto

Es momento de entender y propiciar que los profesores, estudiantes y profesionales de Enfermería deben aprender patrones y prácticas de cuidado humanísticos. Es necesario admitir que el proceso enseñanza - aprendizaje del cuidado, no es un fenómeno que pueda ser heredado, o transmitido fácilmente, sino que debe ser enseñado y aprendido a través de prácticas escolares, de lecturas, escritos, experiencias vividas en conjunto, historias de vida, narrativas, pero sobre todo brincar el velo de la dificultad del aprendizaje del cuidado.
José Luis Coraggio en 1994 reconoce la necesidad de una pedagogía crítica, donde la estrategia fundamental es la reflexión sobre lo que generalmente se toma como dado: el proceso enseñanza - aprendizaje, creando las condiciones más favorables para un aprendizaje efectivo, capaz de transformar a educandos y a educadores ${ }^{7}$.

De acuerdo con las vertientes planteadas por Coraggio, el proceso enseñanza - aprendizaje tiene tres visiones de desarrollo: a) la relación docente - educandos, b) la relación alumno texto y c) la relación alumno contexto. Pero para desarrollar el presente trabajo es que plantearemos alternativas en función de las dos últimas visiones, dirigidas a la enseñanza del cuidado.

La relación alumno - texto tiene un significado trascendente, ya que en los procesos de formación superior, hemos hecho de la lectura, una habilidad implícitamente puesta en el centro de su formación. Las evaluaciones suelen centrarse en el grado de asimilación del discurso científico, y en ocasiones práctico, como es el caso de la práctica del Cuidado de Enfermería.

La propuesta de enseñanza para el cuidado en relación a esta visión de alumno - texto manifestada anteriormente es planteada desde las estrategias de la corriente cognoscitivista retomando el concepto de Aprendizaje Significativo postulado por Ausubel, quien maneja que el aprendizaje implica una reestructuración activa de las percepciones, ideas, conceptos y esquemas que el aprendiz posee en su estructura cognitiva, dice que el aprendizaje es sistemático y organizado por ser un fenómeno complejo que no puede ser reducido a simples asociaciones memorísticas; por el contrario, propugna por el aprendizaje verbal significativo el cual permite el dominio de los contenidos curriculares que se imparten en las escuelas, principalmente en las que se imparte nivel medio y superior ${ }^{8}$.

Incluso José Luis Medina, hace alusión en sus reflexiones acerca de que el conocimiento de Enfermería debe ser significativo; sus planteamientos los hace a partir de la "hermenéutica textual" la cual la conceptúa como el intento de la comprensión durante la aproximación a un texto, que es mediado por una serie de significados, creencias y expectativas previos, los cuales a través de nuestras interpretaciones, interaccionan con el significado que el autor trataba de comunicar, de tal manera que dicho texto nos resulte inteligible, comprensible o significativo.

Hemos de reconocer que los planteamientos curriculares de la disciplina de Enfermería ya no pueden seguir 
encasillados en una concepción bancaria, memorística, pasiva y receptiva del sujeto que aprende. En la idea de hacer significativos los aprendizajes para la enseñanza del cuidado en Enfermería es que retomamos la propuesta de Coll, Pozo, Sarabia y Valls ${ }^{9}$, quienes distinguen que los planteamientos en un plan de estudios pueden ser del tipo de conocimientos "declarativo", "procedimental y "actitudinal - valoral" (ver figura No. 1).

\section{El aprendizaje de contenidos declarativos.}

El "saber que" o conocimiento declarativo ha sido una de las áreas de contenido mas usadas dentro de la formación no solo de niños y adolescentes, sino también en la formación profesional y de posgrado. No se debe demeritar su valor, ya que la mayoría de las asignaturas o cuerpos de conocimientos disciplinar tienen esta característica, ya que constituye por lo menos en lo que a Enfermería respecta, su lucha por edificarse como disciplina (esto desde el punto de vista positivista, que ha predominado aún en la actualidad).

Parece un tanto mecanicista, denominarlo declarativo, sin embargo, se le dio tal posición por que es un saber que se dice, que se declara o que se conforma y expresa por medio del lenguaje. Y dentro del conocimiento declarativo, se expresan dos tipos de ellos:

a) El conocimiento factual, que es el que refiere a datos y hechos que proporcionan información verbal y que el alumno debe de aprender en forma literal o "al pie de la letra"; no se requiere de conocimientos previos y es memorístico. Las estrategias para obtener una memorización significativa y que vincule la información factual entre sí y con otro tipo de contenidos; puede ser por ejemplo con el repaso, la relectura u otras.

b) El conocimiento conceptual, que es más complejo que el anterior, ya que se construye a partir del aprendizaje de conceptos, principios y explicaciones, los cuales ya no es requisito o no debiera ser requisito aprendérselos en forma literal, sino que su importancia para ser significativo, radica en abstraer el significado esencial o identificando las características definitorias y las reglas que lo integran. Su diferencia con el conocimiento radica en que el conocimiento conceptual debe tener una asimilación sobre el significado de la información nueva, se debe comprender lo que se está aprendiendo, donde es preponderante la vinculación con conocimientos previos pertinentes que posee el alumno.
Las estrategias para promoverlo requieren que los materiales de aprendizaje se organicen y estructuren correctamente, lo cual les provee de una riqueza conceptual que pueda ser explotada por los alumnos ${ }^{8}$. Es necesario hacer uso de los conocimientos previos y hacer que éstos impliquen cognitiva, motivacional y efectivamente en el aprendizaje. El docente debe de planear actividades para que los alumnos tengan oportunidades para explorar, comprender y analizar los conceptos de forma significativa, ya sea mediante una estrategia expositiva o por descubrimiento.

En el cuadro No. 1 se describen las estrategias para la enseñanza del conocimiento de tipo factual y conceptual, desde los planteamientos de Díaz y Hernández ${ }^{8}$, con las estrategias propuestas por las autoras para su traspolación a la enseñanza en Enfermería:

\section{El aprendizaje de conocimientos procedimentales}

El "saber hacer" o saber procedimental, se refiere a la ejecución de procedimientos, y manifiesta el dominio de habilidades técnicas, destrezas y formas de realizar situaciones. Donde su principal característica es de ser de tipo práctico, a diferencia del conocimiento declarativo, que era teórico.

Según Colls y Valls ${ }^{9}$, los procedimientos son un conjunto de acciones ordenadas y dirigidas a la consecución de una meta determinada; y que de acuerdo a este último autor, es necesario clarificar al estudiante la meta a lograr, la secuencia de acciones a realizar y la evolución temporal de las mismas.

Díaz y Hernández, han establecido que para este tipo de aprendizaje, se requiere de ciertas etapas, entre ellas:

1. La apropiación de datos relevantes respecto a la tarea y sus condiciones: Por lo tanto, aquí también se hace uso primero del conocimiento declarativo, sin todavía pasar a la ejecución de una tarea. Se da al alumno la información o conocimiento relacionado con el procedimiento general y las tareas puntuales a desarrollar (si es que existen etapas de consecución), explicarle las propiedades y condiciones para su realización, así como las reglas generales de aplicación.

2. La actuación o ejecución del procedimiento, el alumno actúa por ensayo y error, y el docente va retroalimentando en episodios de intervención. Por lo tanto, intervienen contenidos declarativos y procedimentales, lo que procura la fijación del procedimiento. 
3. La automatización del procedimiento, es a partir de su uso o aplicación en situaciones pertinentes. Cuando se sitúa la fijación y realización del procedimiento, se genera facilidad. Ajuste, unidad y ritmo continuo cuando se ejecuta.

4. El perfeccionamiento indefinido del procedimiento, que a base de ejecuciones hacen del estudiante un experto.

Es importante hacer notar, que en el aprendizaje procedimental, además de plantear las vías o rutas adecuadas para realizarlo, también juegan un papel importante, las rutas erróneas y las alternativas u opciones de aplicación y solución de problemas al presentarse éstos. Es importante resaltar que no se trata de una experimentación meramente conductista, sino que las intervenciones del guía (docente), la motivación, el uso de la metacognición (hacerse sensible de conocer el conocimiento) y la autorregulación (control de los procesos en uno mismo de lo que aprende y como lo aprende), es decir, debe imperar una reflexión y análisis continuo sobre las actuaciones, tanto de alumno como de docente.

Díaz y Hernández ${ }^{8}$, proponen los siguientes recursos instruccionales, pero a su lado se hace el planteamiento de la articulación con los aprendizajes procedimentales para Enfermería (Ver Cuadro No. 2).

\section{El aprendizaje de contenidos actitudinal y de valores}

Las actitudes son constructos que median nuestras acciones, que están compuestos de un componente cognitivo, un componente afectivo y un componente conductual ${ }^{9}$. $Y$ que en realidad son experiencias de tipo subjetivo (por implicar cognición y afectividad) que implican juicios evaluativos (análisis del si o no realizarlos, o por qué hacerlo), que son expresados en forma verbal y no verbal, que son relativamente estables y son aprendidos en el CONTEXTO social. Los valores son en realidad el reflejo de los valores que poseemos cada uno.

El aprendizaje de las actitudes y valores es un proceso lento y gradual, e incluso más que los otros tipos de conocimientos (declarativo y procedimental), además de influir en él las experiencias previas personales, las actitudes de otras personas significativas (amigos, docentes, familia, pareja, etc), la información y experiencias novedosas y el contexto sociocultural. Pero por largo tiempo se ha consi- derado ajena esta actividad en la pedagogía, a pesar de que inconscientemente nos encontremos influenciando en todo momento al educando, y muchas veces no en valores y actitudes positivas, sino con antivalores (discriminación, autoritarismo, maltrato, etc).

Se hace uso por lo común de las técnicas participativas, las discusiones y técnicas de estudio activo, las exposiciones y explicaciones de carácter persuasivo (con conferencistas especialistas en el tema); pero el actor principal, en este aprendizaje, son docentes y alumnos. No nos estamos refiriendo al sólo hacer evidentes enunciados declarativos o el indicar que se lleve a la práctica el ejercicio de los valores como la solidaridad o el respeto; sino que se debe incidir significativamente en el comportamiento del alumno, en su capacidad de comprender críticamente la realidad del entorno, el desarrollo de habilidades para el diálogo, la coparticipación y la tolerancia; y sobre todo con el desarrollo de vínculos alumnos - docentes.

A continuación se plantean las estrategias de Díaz Barriga y Hernandez ${ }^{9}$, para la enseñanza de valores y a la derecha su vinculación con la enseñanza en Enfermería. (Ver Cuadro No. 3):

\section{CONCLUSION}

Strickland ${ }^{10}$, enuncia que el cuidado humano es la fuente de nuestra conciencia y se manifiesta en el cuidado ofrecido (practicar el cuidado), como la expresión de nuestra humanidad y reflexión de nuestros valores, principios profesionales y personales. En este sentido, si dentro de la parte del texto, no desarrollamos los vínculos axiológicos como profesionales del cuidado, no esperemos que el estudiante los pueda poner en acción en una práctica que le atemoriza por tener contacto con otros que le son extraños; incluyendo a su docente, compañeros y aún más al sujeto del cuidado.

Concordamos con Medina en que la educación de enfermeras tiene lugar en situaciones sociales de gran complejidad y ambigüedad que no pueden resolverse por la mera aplicación de conocimientos científicos, ya que una actuación práctica es una acción regida deliberadamente por principios éticos ${ }^{11}$. Requiere además de una plataforma informativa (conocimientos), el juicio responsable de los participantes y su compromiso.

Las acciones por las que se lleva a cabo el proceso educativo en Enfermería son acciones de tipo comunicativas $y$, por lo tanto confluyen deseos, intereses, motivaciones, expectativas e interpretaciones de los participantes. Y en el 
momento de que los estudiantes de Enfermería tengan que adecuar y tomar decisiones y dirigir intervenciones, éstos se encontraran lógicamente mediados por los significados que les otorguen los participantes.

Enfermería requiere con urgencia un replanteamiento de su práctica educativa, para desarrollar en los educandos la motivación para aprender, para buscar y asimilar conocimientos, para producir nuevos conocimientos a partir de la reflexión y la observación, es fundamental según Coraggio ${ }^{7}$ interiorizar una siempre insatisfecha necesidad de continuar aprendiendo a través de una práctica continuada y exitosa que demuestre la utilidad de aprender. Tal vez aquí es donde ha estado el vacío en nuestra disciplina, los alumnos no

CUADRO NO. 1

\begin{tabular}{|c|c|c|c|}
\hline $\begin{array}{l}\text { TIPO DE } \\
\text { CONOCIMIENTO }\end{array}$ & $\begin{array}{l}\text { ESTRATEGIAS PARA } \\
\text { PROMOVERLO }\end{array}$ & TECNICA & $\begin{array}{l}\text { EJEMPLIFICACION EN LA ENSEÑANZA } \\
\text { DEL CUIDADO DE ENFERMERIA. }\end{array}$ \\
\hline FACTUAL & $\begin{array}{l}\text { Lectura y relectura. } \\
\text { Organización categorial } \\
\text { Elaboración verbal } \\
\text { y visual }\end{array}$ & $\begin{array}{l}\text { Repetición simple: se repite } \\
\text { varias veces cada término. } \\
\text { Repetición parcial: se repiten } \\
\text { juntos grupos de términos. } \\
\text { Repetición acumulativa: se } \\
\text { añade otro término más a los } \\
\text { de la vez anterior. } \\
\text { Consiste en agrupar los nom- } \\
\text { bres en función de categorías } \\
\text { de pertenencia. } \\
\\
\text { Crear una frase en la que apa- } \\
\text { rezca el término o términos a } \\
\text { aprender, o en crear una ima- } \\
\text { gen que facilite su asociación. }\end{array}$ & $\begin{array}{l}\text { Para la enseñanza de las características que se contem- } \\
\text { plan en la Valoración de Capurro en el recién nacido, una } \\
\text { lectura acerca de los parámetros que se evalúan en dicho } \\
\text { examen, luego en orden significativo para el alumno orga- } \\
\text { nizarlo, para que pueda aprehenderlo. } \\
\text { Por ejemplo en el aprendizaje de los tipos de aislamiento } \\
\text { en caso de enfermedades infecciosas, éstos se organiza- } \\
\text { rán por las formas en que se diseminan los organismos } \\
\text { patógenos o el aparato o sistema afectado en el individuo } \\
\text { en aislamiento (gota, via aérea y contacto). } \\
\text { Por ejemplo, si el término a aprender es diálisis peritoneal, } \\
\text { éste se introduce en un texto, por ejemplo un caso clínico } \\
\text { que muestre incluso el objetivo de aplicarla, de tal forma, } \\
\text { que el estudiante, no sólo en forma conceptual obtenga el } \\
\text { significado, sino además lo visualice desde planteamientos } \\
\text { del porqué se llevó a cabo tal procedimiento (en la lectura) } \\
\text { y deduzca por tanto el significado. }\end{array}$ \\
\hline & $\begin{array}{l}\text { Selección (es un apoyo } \\
\text { al repaso) }\end{array}$ & $\begin{array}{l}\text { Subrayar: marcar con color, las } \\
\text { ideas relevantes y parafrasear } \\
\text { con otro compañero o el do- } \\
\text { cente para ver los puntos de } \\
\text { coincidencia. } \\
\text { Copiar: se trata de rescatar las } \\
\text { ideas principales del texto, o } \\
\text { del concepto. } \\
\text { Anotaciones o formular pre- } \\
\text { guntas: escribir en forma } \\
\text { declarativa o en forma de pre- } \\
\text { guntas breves o reflexiones, } \\
\text { cuestiones sobre el texto, lo } \\
\text { que facilita el vínculo de lo di- } \\
\text { cho con otros puntos. }\end{array}$ & $\begin{array}{l}\text { La lectura del procedimientos de cuidados al paciente } \\
\text { con traqueostomía, se habrán se subrayar las palabras o } \\
\text { ideas relevantes (ej. Traqueostomía, secreción, aspiración, } \\
\text { ventilación, etc). Se le pedirá al alumno escriba preguntas } \\
\text { concretas que le ocasionó la lectura. Hará enunciados pa- } \\
\text { rafraseados con las ideas centrales por ejemplo del objeti- } \\
\text { vo de este procedimiento o los puntos fundamentales para } \\
\text { llevarlo a cabo. }\end{array}$ \\
\hline
\end{tabular}

aprenden, memorizan e incluso hasta expresan que lo que vivieron en la escuela no fue útil para su desarrollo en el plano laboral.

Es por medio de la comunicación y relación estrecha, que el docente puede trasmitir a sus estudiantes el amor por la Enfermería, y la responsabilidad humana de cuidar. El uso del aprendizaje significativo en el uso del texto y del contexto, en conjunción de la experiencia vivida y compartida de las situaciones de Enfermería, pueden hacer del cuidado esa práctica exitosa y satisfactoria para los futuros profesionales de la Enfermería, así como para los docentes que los forman.

Estrategias para promover el aprendizaje factual del Cuidado de Enfermería 


\begin{tabular}{|l|l|l|l|}
\hline CONCEPTUAL & $\begin{array}{l}\text { Estrategia expositiva } \\
\text { Procesamiento simple } \\
\text { (obtener palabras clave, } \\
\text { imágenes mentales, pa- } \\
\text { rafraseo. }\end{array}$ & $\begin{array}{l}\text { Representación gráfica de re- } \\
\text { des conceptuales (mapas con- } \\
\text { ceptuales), su beneficio es que } \\
\text { además de presentar las rela- } \\
\text { ciones entre conceptos, mani- } \\
\text { fiesta relaciones jerárquicas. }\end{array}$ & $\begin{array}{l}\text { Nuevamente para el ejemplo de medidas de protección } \\
\text { contra enfermedades infecciosas, se puede elaborar un } \\
\text { mapa conceptual en el que la cima sea representada por } \\
\text { las medidas generales y subsiguiente hacia abajo y en el } \\
\text { mismo nivel los tipos de aislamiento. }\end{array}$ \\
\hline Descubrimiento & $\begin{array}{l}\text { Investigación. } \\
\text { Elaboración de significados } \\
\text { a partir de conceptos clave en } \\
\text { lecturas previas. }\end{array}$ & $\begin{array}{l}\text { Se pregunta acerca de las experiencias previas entre los } \\
\text { alumnos, al estar enfermos de diarrea, cuales han sido las } \\
\text { manifestaciones o expresiones sintomáticas y lo que han } \\
\text { hecho o hicieron en su momento como medida correctora; } \\
\text { lo que hará significativo para ellos la rehidratación oral, y } \\
\text { aún más en caso de infantes. } \\
\text { Se les pide a los alumnos investigar el tema de rehidratación } \\
\text { oral, de diversas fuentes. } \\
\text { O se dan textos en que se hable de la rehidratación, } \\
\text { cuidando de no incluir el significado, para que posterior a } \\
\text { la lectura y en equipo o parejas se elaboren éstos. }\end{array}$ \\
\hline
\end{tabular}

CUADRO NO. 2

Estrategias para el aprendizaje procedimental en Enfermería

\section{ESTRATEGIA PARA APRENDIZAJE PROCEDIMENTAL}

Repetición y ejercitación reflexiva.

Imitación a modelos apropiados

Observación crítica

Retroalimentación oportuna, pertinente y profunda.

Establecimiento del sentido de las tareas y del proceso en su conjunto, mediante la evocación de conocimientos y experiencias previos.

Verbalización mientras se aprende.

Actividad intensa del alumno, centrada en condiciones auténticas, lo más cercanas y reales a las condiciones reales donde se aplica lo aprendido.

Fomento de la metacognición: conocimiento, control y análisis de los propios comportamientos.

\section{ESTRATEGIA DE APRENDIZAJE PROCEDIMENTAL PARA ENFERMERÍA (Maniobras de Leopold).}

Tomemos de ejemplo, el aprendizaje de las maniobras de Leopold, cuyo objetivo es poder localizar la situación, posición, etc del producto dentro del útero materno. Se hace uso del aprendizaje declarativo, una y otra vez, rescatando los puntos trascendentes como la importancia de las maniobras, la observación (uso de los cinco sentidos), la atención consiente y profunda del alumno, etc.

Demostraciones sistemáticas, pausadas, con enunciados declarativos, el uso de conectores verbales que resalten la importancia de tal o cual paso, etc.

Al solicitarle que trate de actuar, por ejemplo en un maniquí, no tacharlo, sino retroalimentarlo (decirle, recuerda, tu objetivo es $\mathrm{x}$, reconsidera, etc).

La retroalimentación debe ser en el momento que observamos una desviación o que el alumno nos pide ayuda, e incluso la intervención por parte del docente no debe ser peyorativa, o con el afán de hacer notar errores, y mucho menos, tratándose de la presencia del paciente, demás personal del equipo de salud, etc. Esta retroalimentación lo que debe otorgar es confianza y por tanto seguridad en el estudiante en futuras ejecuciones.

El hablar de las maniobras de Leopold, no es un procedimiento aislado, pertenece a la Valoración prenatal, y está al Cuidado del Trinomio padre - madre - hijo. Se contextualiza en relación a los embarazos, que el estudiante ha visto, experimentado o participado por ser un familiar cercano. Estas vinculaciones hacen significativo el aprendizaje.

Para muchos estudiantes, es una estrategia, el pedirle que verbalice mientras actúa, ya que así va haciendo en forma profunda y consiente el procedimiento, incluso también concientiza sus dificultades, o errores.

Debiera ser la ejercitación de las maniobras de Leopold, en una mujer embarazada, para que en realidad observe y sienta, para aprender significativamente en su contacto por ejemplo con esa parte de la realidad del Cuidado en el trinomio padre- madre- hijo.

Nunca dejar de insistir y motivar al alumno para la reflexión de lo que se encuentre ejecutando, y hacerlo consiente de que sea congruente de lo que piensa, verbaliza y ejecuta. 
CUADRO NO. 3

Estrategias para el aprendizaje de valores en el cuidado de Enfermería

\begin{tabular}{|l|l|r|}
\hline ESTRATEGIAS & CARACTERISTICAS & $\begin{array}{c}\text { TRASPOLACION A LA ENSEÑANZA } \\
\text { DEL CUIDADO DE ENFERMERIA }\end{array}$
\end{tabular}

- Orientado al autoconocimiento y análisis crítico de la realidad personal, familiar, comunitaria.

Clarificación de valores y juicio crítico
- Se basa en preguntas esclarecedoras, reflexión personal, hojas de trabajo e instrumentos de autoanálisis, y elaboración de proyectos y planes.
- Dirigir preguntas, para elaborar ensayos acerca de que sienten al ser enfermeros, como se visualizan en su ayuda a sí mismos, a la familia y la comunidad

- Llevar a cabo un análisis de las características de los profesionales de Enfermería y la razón de ellas.
Discusión de dilemas y análisis de casos.

\begin{tabular}{|l} 
\\
\hline \\
Comprensión y \\
escritura crítica \\
de "texto".
\end{tabular}

Aprendizaje
cooperativo y
"situado" orien-
tado a

la comunidad.

- Promueve no sólo el trabajo en equipo, sino la vivencia de valores como la solidaridad, ayuda mutua, responsabilidad conjunta, empatía, ética profesional, etc.

- Fomenta una labor social de apoyo y servicio a la comunidad circundante mediante el desarrollo y operación de proyectos de intervención social o profesional. cuestiones sociales, cívicas, personales, con implicaciones éticas.

- Promueve procesos de identificación, empatía, razonamiento y toma de decisiones en torno a situaciones que se enfrentan social y cotidianamente.

- Intenta el desarrollo de una moral.

- Analiza y produce "textos" (escritos, películas, canciones, fotografías, etc).

- Buscar diversas fuentes de información con distintas posturas.

- Fomenta competencias comunicativas básicas, orales y escritas.
- Plantear situaciones de Enfermería, en que se de a conocer las experiencias particulares del vínculo de enfermero - paciente, en las cuales se trascienden los valores.

- Pedir la elaboración de sociodramas con temas que impliquen el plantear la expresión de sus sentimientos, tales como el aborto, la drogadicción, la eutanasia, la muerte, la enfermedad crónica, etc. Y al final recapitular la importancia de los valores en estos temas.
- Pedir la elaboración de ensayos respecto a vivencias propias de los alumnos que impliquen su relación moral y/o ética en la p'ractica cxotidiana, o en su vida familiar.

- Ante temas de debate como el aborto, la eutanasia, etc, pedir que indaguen diversas fuentes, para hacer la revisión de posturas éticas descritas en diversas fuentes.
- Promover la participación comunitaria en campañas de vacunación infantil, antirrábica, etc.

- En situaciones de desastre promover la participación comunitaria de grupos de Enfermería, donde sean congruentes el grado de preparación (semestre), con el tipo de actividad supervisada que se requiera en la comunidad.

- Promover la participación en cuerpos colegiados, o asociaciones de profesionales de Enfermería o asociaciones interdisciplinarias con propósitos legales.
Desarrollo de Habilidades sociales, afectivas y de autoregulación.
- $\quad$ Planear estrategias para el manejo de emociones y sentimientos, conducta prosocial, asertividad, solución de problemas, realización de planes de vida personales y autocompromiso.

- Enfatizar las habilidades para el diálogo, la comprensión crítica de la realidad, la tolerancia, la autodirección y la participación activa.
- Fomentar el ejercicio escrito de elaboración de planes de vida, en función de su identidad profesional de Enfermería.

- Elaborar metas a corto, mediano y largo plazo acerca de otros niveles de estudio, lugar donde desea situarse laboralmente.

- Ofrecer las alternativas laborales en cuanto a Enfermería avanzada (lugares donde la Enfermería incursiona y tiene un papel relevante no sólo prácticamente, sino en el ámbito ético - legal). 


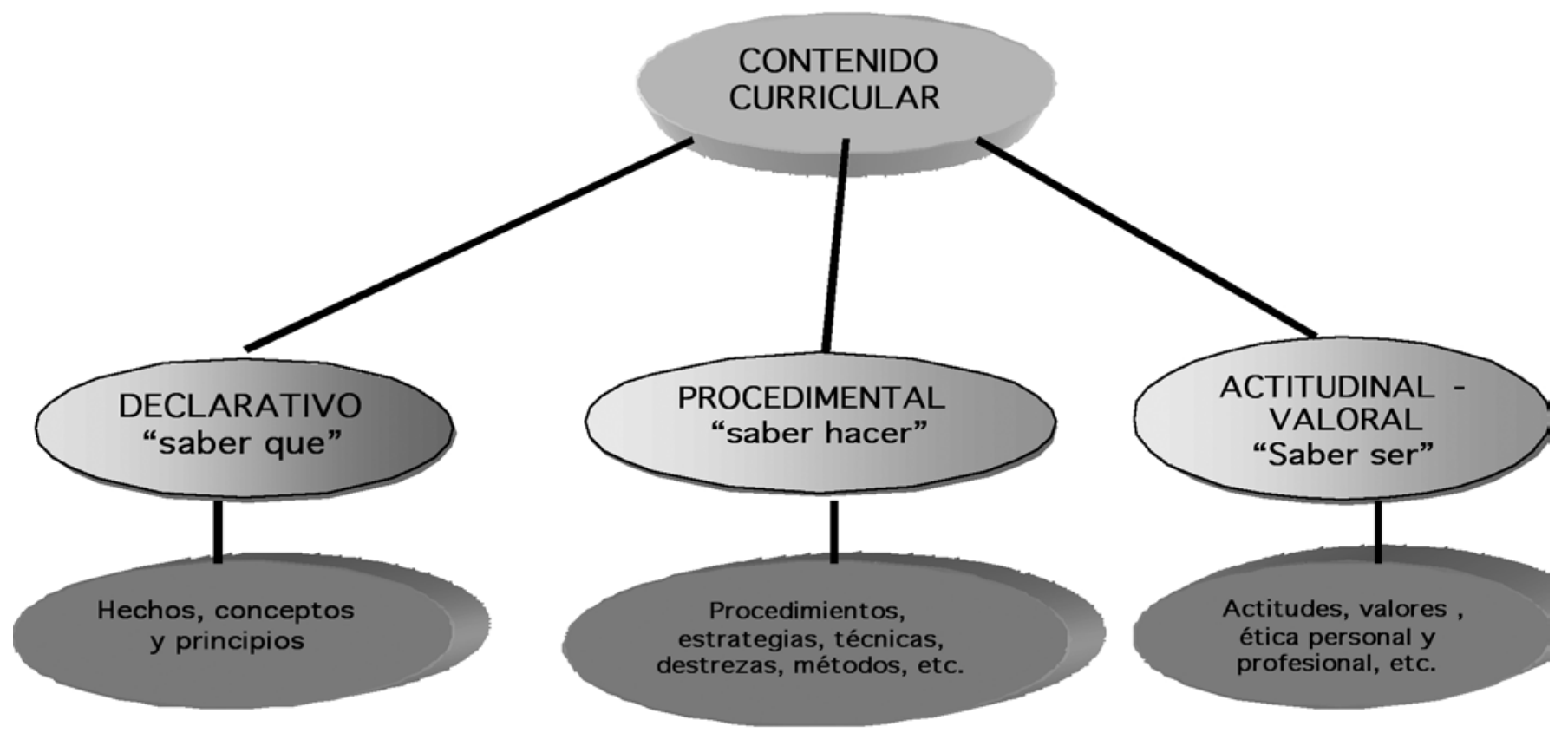

Fig. 1. Los contenidos curriculares. Tomado de Díaz Barriga y Hernández R.

\section{REFERENCIAS BIBLIOGRAFICAS}

1 Moreno, Y., Muñoz, S., \& Alvis, T. Empoderamiento en el cuidado. En: GRUPO DE CUIDADO. El Arte y la Ciencia del Cuidado. Universidad Nacional de Colombia: Colombia. (2002). p. 321

2 Wright, G. Explicación y comprensión. En: GRUPO DE CUIDADO. El Arte y la Ciencia del Cuidado. Universidad Nacional de Colombia: Colombia. (2002). p. 129

3 Gonzalez HE. Implicaciones del cuidado. En: GRUPO DE CUIDADO. El Arte y la Ciencia del Cuidado. Universidad Nacional de Colombia: Colombia. (2002). p.43

4 Centeno, R y Col. Análisis de los intereses de la Vida Cotidiana y Ciencias. En: GRUPO DE CUIDADO. El Arte y la Ciencia del Cuidado. Universidad Nacional de Colombia: Colombia. (2002) p. p.21

5 Medina, JL.. La pedagogía del cuidado: saberes y prácticas en la formación universitaria en Enfermería. Laertes/Psicopedagogía: Barcelona, España. (1998) p.54

6 Pinto, A. El cuidado en el Proceso de Enseñanza Aprendizaje. (En: GRUPO DE CUIDADO. El Arte y la Ciencia del Cuidado. Universidad Nacional de Colombia: Colombia. (2002). p. 45

7 Coraggio, J. Pedagogía crítica: Eje de desarrollo de la enseñanza superior. Trabajo preparado para el Rectorado de la Universidad Nacional de General Sarmiento: Buenos Aires. (1994). p.231
8 Díaz- Barriga, AF. \& Hernández, RG. Estrategias docentes para un aprendizaje significativo: una interpretación constructivista. Ed. Mc Graw Hill - Interamericana: México. (2002). p. 178

9 Coll, JI., Pozo, B. Sarabia y Valls, E. Los contenidos de la reforma. Enseñanza y aprendizaje de conceptos, procedimientos y actitudes. En : DÍAZ- BARRIGA, A. F. \& HERNÁNDEZ, R. G. Estrategias docentes para un aprendizaje significativo: una interpretación constructivista. Ed. Mc Graw Hill Interamericana: México. (2002). p.237

10 Strickland, G. (1996). Experiencia de recibir un cuidado de Enfermería humanizado en un servicio de hospitalización. En: GRUPO DE CUIDADO. El Arte y la Ciencia del Cuidado. Universidad Nacional de Colombia: Colombia. (2002). p.79

11 Angulo, JF. El problema de la credibilidad y el lugar de la triangulación en la investigación interpretativa: un análisis metodológico. En: MEDINA, JL. La pedagogía del cuidado: saberes y prácticas en la formación universitaria en Enfermería. Laertes/Psicopedagogía: Barcelona, España. (1998) p.54

\section{Dirección para correspondencia}

Mtra. María Lidia Bernal B.: marthaliliabb@hotmail.com Mtra. Gandhy Ponce Gómez: gandhy_ponce@yahoo.com.mx 